\title{
Mortalidad en hospitalizados con traumatismo torácico: variables asociadas, causas y distribución temporal
}

\author{
Roberto González L. ${ }^{1,2}$, Alejandra Riquelme U. ${ }^{3}$, Rodrigo Reyes M. ${ }^{1,2}$,
} Sebastián Barra M. ${ }^{4}$, Felipe Alarcón O. ${ }^{4}$, Enrique Seguel S. ${ }^{1,2}$, Aleck Stockins L. ${ }^{1,2}$, Andrés Jadue T. ${ }^{1}$, Andrés Schaub C. ${ }^{4}$, Diego Saldivia Z. ${ }^{4}$ y Emilio Alarcón C. ${ }^{1,2}$

${ }^{1}$ Centro Cardiovascular, Hospital Clínico Regional Dr. Guillermo Grant Benavente. Concepción, Chile. Departamento de Cirugía, Facultad de Medicina, Universidad de Concepción. Concepción, Chile. Servicio de Cirugía Hospital Clínico Regional Dr. Guillermo Grant Benavente. Concepción,

${ }^{4}$ Facultad de Medicina, Universidad de Concepción. Concepción, Chile.

Esta comunicación orresponde a uno de los análisis de la base de datos prospectiva de

hospitalizaciones por traumatismo torácico iniciad el año 1981 en Concepción,

Recepción 2020-09-02 aceptado 2020-10-08

Correspondencia a: Dr. Roberto González L. rgonzalezlagos@udec.cl

\section{Associated variables, causes and time distribution of mortality in thoracic trauma patients}

Background: Trauma is one of the ten leading causes of death worldwide and the first among the youth. Thoracic trauma (TT) is present in a high percentage of deaths due to trauma and is the second leading cause of death after traumatic brain injury. Aim: To analyze the mortality associated variables, major causes and temporal distribution of mortality among dead hospitalized patients with TT. Materials and Method: Observational study in hospitalized patients with TT, period January 1981 to December 2018. Review of prospective TT protocols and data base. Major syndromic causes of death were recorded and a logistic regression for variables associated with mortality was made. SPSS $25^{\circledR}$ with chi-quadrat tests was used to compare classification, type of TT and temporal distribution. A p value $<0,05$ was considered significant. Results: Total 4.297 TT and global mortality was $120(2,8 \%)$ cases. The main independent variables associated with mortality were the physiological decline upon admission, massive hemothorax and TT by firearms. The leading cause of death was hypovolemic shock, with significant differences according to the type of TT in the first 4 and 24 hours. In the temporal distribution was observed that, the deaths with penetrating and isolated TT were earlier and that there was no second peak of mortality following the first week. Conclusions: Independent variables associated with mortality were observed among hospitalized patients with TT, being physiological deterioration the most important factor. Besides, there are significant differences in the death causes and temporal distribution of mortality among the different subgroups of hospitalized patients with TT.

Key words: wounds and injuries; thoracic injuries; mortality; trauma severity indices; thoracic surgery.

\section{Resumen}

Introducción: Los traumatismos están entre las diez principales causas de muerte a nivel mundial y son la primera en jóvenes. El traumatismo torácico (TT) está presente en un alto porcentaje de las muertes por traumatismos y es la segunda causa de muerte después del traumatismo encefalocraneano. Objetivos: Analizar las variables asociadas a mortalidad, las causas principales y la distribución temporal de la mortalidad en hospitalizados fallecidos con TT. Materiales y Método: Estudio observacional de hospitalizados con TT, período enero de 1981 a diciembre de 2018. Revisión de protocolos prospectivos de TT y base de datos. Se consignaron las causas de muerte sindromáticas principales y se realizó una regresión logística para variables asociadas a mortalidad. Se utilizó SPSS $25^{\circledR}$ con pruebas chi-cuadrado para comparar clasificación, tipo de TT y su distribución temporal, considerando significativo $\mathrm{p}<0,05$. Resultados: Total 4.297 TT, mortalidad global de 120 (2,8\%) casos. Las principales variables independientes asociadas a mortalidad fueron el deterioro fisiológico al ingreso, el hemotórax masivo y el TT por arma de fuego. La principal causa de muerte fue el shock hipovolémico, con diferencias significativas según tipo de TT en las primeras 4 y 24 horas. En la distribución temporal se observó que las muertes con TT penetrante y aislado fueron más precoces y no se evidenció un nuevo peak 
en la mortalidad luego de la primera semana. Conclusiones: Se observaron variables independientes asociadas a mortalidad en hospitalizados con TT, siendo el deterioro fisiológico al ingreso el factor más importante. Además, existen diferencias significativas en las causas de muerte y distribución temporal de la mortalidad entre diferentes subgrupos de hospitalizados con TT.

Palabras clave: heridas y traumatismos; traumatismos torácicos; mortalidad; puntaje de gravedad del traumatismo; cirugía torácica.

\section{Introducción}

Los traumatismos están entre las diez principales causas de muerte a nivel mundial y son la primera en jóvenes, representando una alta carga económica por hospitalización y muerte prematura ${ }^{1,2}$.

El traumatismo torácico (TT) representa el 10\%$15 \%$ de todos los traumatismos y se encuentra en aproximadamente la mitad de los politraumatizados. Además, corresponde a la segunda causa de muerte después del traumatismo encefalocraneano (TEC) y se estima que contribuye de forma significativa en la mortalidad en al menos otro $25 \%-50 \%$ de los traumatismos ${ }^{3-5}$.

Diferentes estudios han detallado los factores principales que determinan la muerte luego de un traumatismo, así como su etiología y temporalidad, sin embargo, existen escasas publicaciones que describan estas características en los fallecidos con $\mathrm{TT}^{6,7}$

Nuestros objetivos son analizar las variables asociadas a mortalidad, las causas principales y la distribución temporal de la mortalidad en hospitalizados fallecidos con TT.

\section{Materiales y Método}

Estudio observacional de hospitalizados con TT en el Hospital Clínico Regional "Dr. Guillermo Grant Benavente" de Concepción, Chile, tratados por el equipo de Cirugía Cardiotorácica entre enero de 1981 y diciembre de 2018.

La información se obtuvo a partir de protocolos prospectivos de TT realizados al momento del ingreso hospitalario, bases de datos, protocolos quirúrgicos y fichas clínicas. Se calcularon los índices de gravedad de traumatismo (IGT): Injury Severity Score (ISS) ${ }^{8}$, Revised Trauma Score Triage (RTS$\mathrm{T})^{9}$ y Trauma Injury Severity Score (TRISS) ${ }^{10,11}$. El ISS determina la gravedad de acuerdo con la lesión anatómica, el RTS-T incluye variables fisiológicas como la presión arterial sistólica, frecuencia respiratoria y escala de Glasgow y el TRISS pondera los dos IGT anteriores y los relaciona con la edad del paciente y el mecanismo del traumatismo, determinando el riesgo de mortalidad. Se consideró como politraumatizado un ISS $>16$ puntos $^{12}$.

A modo de facilitar el análisis se definieron grupos etarios de acuerdo con la Organización Mundial de la Salud ${ }^{13,14}$; se consignó la causa de muerte sindromática y se agruparon las muertes producidas por el síndrome de respuesta inflamatoria sistémica (SIRS) y la falla orgánica múltiple (FOM) en una misma categoría, utilizando las definiciones aceptadas internacionalmente ${ }^{15,16}$.

Se tabularon los datos en planilla de Microsoft Excel $^{\circledR}$ y se realizó análisis estadístico con el programa $S P S S 25^{\circledR}$, con función chi-cuadrado para las variables categóricas. Se realizó una regresión logística con cálculo de Odds Ratio (OR) para determinar variables independientes asociadas a mortalidad, considerando un intervalo de confianza del 95\% y como significativo un valor $\mathrm{p}<0,05$.

Se describieron las variables asociadas a mortalidad, así como el número, sexo, clasificación, mecanismo, agente, tipo, lesiones asociadas e IGT de pacientes hospitalizados fallecidos con TT; se compararon las causas de muerte y la distribución temporal de la mortalidad según clasificación o tipo del TT y se graficó la mortalidad acumulada a 30 días de estos grupos.

El estudio y análisis de las hospitalizaciones por TT fue aprobado por el comité ético-científico de nuestra institución.

\section{Resultados}

Durante el período se hospitalizaron 4.306 pacientes con TT, de los cuales se excluyeron nueve por corresponder a menores de diez años, resultando en un total de 4.297 pacientes. De estos, la mortalidad global fue de 120 (2,8\%) casos.

Las principales características generales de los TT, así como la mortalidad global y en grupos seleccionados de la serie se describen en la Tabla 1. Se observó mortalidad en el $5,0 \%$ de los adultos 
Tabla 1. Principales características generales y mortalidad observada en subgrupos seleccionados en relación con el total de hospitalizados con traumatismo torácico

\begin{tabular}{|c|c|c|}
\hline & $\mathbf{n}$ & Mortalidad \\
\hline Total & 4.297 & $120 \quad(2,8 \%)$ \\
\hline \multicolumn{3}{|l|}{ Grupo etario } \\
\hline Adulto mayor & $544(12,7 \%)$ & $27(5,0 \%)$ \\
\hline Adulto & $3.145(73,2 \%)$ & $83(2,6 \%)$ \\
\hline Adolescente & $608(14,1 \%)$ & $10(1,6 \%)$ \\
\hline \multicolumn{3}{|l|}{ Sexo } \\
\hline Mujer & $491(11,4 \%)$ & $16(3,3 \%)$ \\
\hline Hombre & $3.806(88,6 \%)$ & $104(2,7 \%)$ \\
\hline \multicolumn{3}{|l|}{ Clasificación } \\
\hline Contuso & $1.816(42,3 \%)$ & $72(4,0 \%)$ \\
\hline Penetrante & $2.481(57,7 \%)$ & $48 \quad(1,9 \%)$ \\
\hline \multicolumn{3}{|l|}{ Agentes principales } \\
\hline Arma de fuego & $205 \quad(4,8 \%)$ & $14(6,8 \%)$ \\
\hline Vehículo motorizado & $902(21,0 \%)$ & $55(6,1 \%)$ \\
\hline Arma blanca & $2.326(54,1 \%)$ & $32(1,4 \%)$ \\
\hline \multicolumn{3}{|l|}{ Tipo } \\
\hline Politraumatismo & $747(17,4 \%)$ & $76(10,2 \%)$ \\
\hline TT con lesiones extratorácicas & $1.175(27,3 \%)$ & $81 \quad(6,9 \%)$ \\
\hline TT aislado & $3.122(72,7 \%)$ & $39(1,3 \%)$ \\
\hline \multicolumn{3}{|c|}{ Hallazgos y lesiones torácicas principales } \\
\hline Lesión de grandes vasos & $93(2,2 \%)$ & $12(12,9 \%)$ \\
\hline Penetrante cardíaca & $226 \quad(5,3 \%)$ & $28(12,4 \%)$ \\
\hline Hemotórax masivo & $140 \quad(3,3 \%)$ & $17(12,1 \%)$ \\
\hline Tratamiento invasivo & $3.037(70,7 \%)$ & $91(3,0 \%)$ \\
\hline Pleurotomía & $2.252(52,4 \%)$ & $38(1,7 \%)$ \\
\hline Cirugía & $785(18,3 \%)$ & $53(6,8 \%)$ \\
\hline Tratamiento médico exclusivo & $1.260(29,3 \%)$ & $29(2,3 \%)$ \\
\hline
\end{tabular}

TT: Traumatismo torácico. fallecidos con TT, la mediana de edad correspondió a 36 años y fueron en su mayoría hombres $(86,7 \%)$. El traumatismo se clasificó como contuso en 72 $(60,0 \%)$ casos debiéndose principalmente a accidentes de tránsito en vehículos motorizados. Además, existieron lesiones extratorácicas en $81(67,5 \%)$ casos (Tabla 4).

Las lesiones y/o hallazgos torácicos más frecuentemente encontradas encontradas en pacientes fallecidos con TT fueron el hemotórax y las fracturas costales con $83(69,2 \%)$ y $62(51,7 \%)$ casos respectivamente. Junto a esto, las lesiones extratorácicas más comunes fueron en orden de frecuencia: las craneoencefálicas, abdominales y de extremidades (Tabla 5).

Las principales causas de muerte de los fallecidos con TT fueron el shock hipovolémico en 52 (43,3\%) casos y el SIRS/FOM en 37 (30,8\%). Se observaron diferencias en las causas de muerte al comparar según la distribución temporal de la mortalidad, predominando el shock hipovolémico en los fallecidos durante los primeros siete días (Tabla 6).

El 65,8\% de los fallecidos con TT lo hicieron en los primeros siete días de hospitalización, destacándose que en $22(18,3 \%)$ casos la muerte se produjo en las primeras $4 \mathrm{~h}$ y en $34(28,3 \%)$ casos en las primeras $24 \mathrm{~h}$ desde el ingreso (Tabla 7 y Figura 1).

Existieron diferencias estadísticamente significativas en la distribución temporal de la mortalidad al comparar el TT contuso versus el TT penetrante. El 81,3\% de los casos fallecidos con TT penetrante ocurrieron en los primeros siete días, proporción significativamente mayor comparado con el 55,6\% de los contusos $(\mathrm{p}=0,003)$. Similar relación se observó en las muertes durante las primeras 4 y $24 \mathrm{~h}$, ambas con $\mathrm{p}<0,001$ (Tabla 8 y Figura 2A).

En la mayoría de los casos de fallecidos con TT aislado la causa de muerte fue el shock hipovolémico, mientras que en TT con lesiones extratorácicas fueron más frecuentes debido al SIRS/FOM, ambos con $\mathrm{p}<0,05$. Respecto a la distribución temporal de la mortalidad, se observaron muertes más precoces en el TT aislado, durante las primeras 4 y $24 \mathrm{~h}$, siendo significativamente superiores al TT con lesiones extratorácicas. Destacó que en los fallecidos con TT con lesiones extratorácicas, en $77(95,1 \%)$ casos las lesiones torácicas se consideraron como graves o muy graves según ISS (Tabla 9 y Figura 2B).

La mortalidad acumulada, en los primeros 30 días, según la clasificación y el tipo del TT se muestran en las Figuras 3 y 4 respectivamente. En el TT contuso, la mortalidad acumulada se caracterizó por presentarse en forma más tardía y con un aumento constante y sostenido hasta el día 20, determinando 
Tabla 2. Variables asociadas a mortalidad en el análisis univariado en hospitalizados fallecidos con traumatismo torácico

\begin{tabular}{|c|c|c|}
\hline Análisis univariado & $\mathbf{p}$ & Odds Ratio (IC 95\%) \\
\hline Edad $>60$ años & $<0,001^{*}$ & $2,09(1,34-3,26)$ \\
\hline TT contuso & $<0,001^{*}$ & $2,08(1,43-3,01)$ \\
\hline Arma de fuego & $<0,001^{*}$ & $2,76(1,55-4,91)$ \\
\hline Taponamiento cardíaco & $<0,001^{*}$ & $7,08(4,23-11,84)$ \\
\hline Penetrante cardíaca & $<0,001^{*}$ & $6,12(3,92-9,57)$ \\
\hline Hemotórax masivo & $<0,001^{*}$ & $5,45(3,16-9,38)$ \\
\hline Lesión diafragmática & $0,019 *$ & $2,26(1,12-4,54)$ \\
\hline Lesión de parénquima pulmonar & $0,020^{*}$ & $2,34(1,11-4,90)$ \\
\hline Tórax volante & $<0,001^{*}$ & $4,20(2,54-6,94)$ \\
\hline$>6$ costillas fracturadas & $<0,001^{*}$ & $3,71(2,25-6,11)$ \\
\hline Fractura de costillas altas $\left(1^{\mathrm{a}}-2^{\mathrm{a}}\right)$ & $0,003 *$ & $2,18(1,29-3,70)$ \\
\hline Shock & $<0,001^{*}$ & $12,56(8,56-18,41)$ \\
\hline TEC grave & $<0,001 *$ & $5,91(4,04-8,65)$ \\
\hline Necesidad de transfusión & $<0,001^{*}$ & $4,94(3,30-7,40)$ \\
\hline Politraumatizado & $<0,001^{*}$ & $9,00(6,15-13,16)$ \\
\hline RTS-T $<11$ & $<0,001^{*}$ & $26,82(17,87-40,25)$ \\
\hline Lesión de grandes vasos & $<0,001^{*}$ & $5,44(2,83-10,43)$ \\
\hline ISS $\geq 25$ & $<0,001^{*}$ & $18,06(11,98-27,25)$ \\
\hline Necesidad de ventilación mecánica & $<0,001^{*}$ & $13,91(9,46-20,45)$ \\
\hline Cirugía torácica & $<0,001^{*}$ & $3,59(2,48-5,19)$ \\
\hline Cirugía urgente & $<0,001^{*}$ & $6,12(4,20-8,90)$ \\
\hline Cirugía $\leq 24$ h & $<0,001 *$ & $5,59(3,85-8,13)$ \\
\hline
\end{tabular}

IC: Intervalo de confianza; TT: Traumatismo torácico; TEC: Traumatismo encefalocraneano; RTS-T: Revised Trauma Score Triage; ISS: Injury Severity Score. *Estadísticamente significativo.

Tabla 3. Variables independientes asociadas a mortalidad en el análisis multivariado en hospitalizados fallecidos con traumatismo torácico

\begin{tabular}{|lcc|}
\hline Análisis multivariado & $\boldsymbol{p}$ & Odds Ratio (IC 95\%) \\
Arma de fuego & $0,012^{*}$ & $2,80(1,25-6,24)$ \\
Hemotórax masivo & $0,003^{*}$ & $2,85(1,43-5,66)$ \\
TT contuso & $0,004^{*}$ & $2,65(1,36-5,17)$ \\
Politraumatizado & $0,006^{*}$ & $2,40(1,28-4,49)$ \\
Shock & $<0,001^{*}$ & $2,75(1,67-4,52)$ \\
Penetrante cardíaca & $0,025^{*}$ & $2,57(1,12-5,86)$ \\
RTS-T < 11 & $<0,001^{*}$ & $5,44(3,31-8,92)$ \\
ISS $\geq 25$ & $0,016^{*}$ & $2,09(1,14-3,83)$ \\
Necesidad de ventilación mecánica & $<0,001^{*}$ & $2,38(1,50-3,76)$ \\
\hline
\end{tabular}

IC: Intervalo de confianza; TT: Traumatismo torácico; RTS-T: Revised Trauma Score Triage; ISS: Injury Severity Score. *Estadísticamente significativo.
Tabla 4. Edad, sexo, clasificación, mecanismo, agente y tipo del traumatismo en hospitalizados fallecidos con traumatismo torácico

\begin{tabular}{|c|c|}
\hline Total de fallecidos & $120(100 \%)$ \\
\hline \multicolumn{2}{|l|}{ Edad (años) } \\
\hline Promedio & $41,3 \pm 19,4$ \\
\hline Mediana & 36 \\
\hline \multicolumn{2}{|l|}{ Sexo } \\
\hline Hombre & $104(86,7 \%)$ \\
\hline Mujer & $16(13,3 \%)$ \\
\hline \multicolumn{2}{|l|}{ Clasificación } \\
\hline Contuso & $72(60,0 \%)$ \\
\hline Penetrante & $48(40,0 \%)$ \\
\hline \multicolumn{2}{|l|}{ Mecanismo } \\
\hline Accidente de tránsito & $55(45,8 \%)$ \\
\hline Atropello & $34(28,3 \%)$ \\
\hline Colisión o volcamiento & $21(17,5 \%)$ \\
\hline Agresión & $44(36,7 \%)$ \\
\hline Accidente doméstico & $7(5,8 \%)$ \\
\hline Iatrogénico & $4(3,3 \%)$ \\
\hline Accidente laboral & $3(2,5 \%)$ \\
\hline Autoagresión & $2(1,7 \%)$ \\
\hline Otros & $5(4,2 \%)$ \\
\hline \multicolumn{2}{|l|}{ Agente } \\
\hline Vehículo motorizado & $55(45,8 \%)$ \\
\hline Arma blanca & $32(26,7 \%)$ \\
\hline Arma de fuego & $14(11,7 \%)$ \\
\hline Objeto contundente & $12(10,0 \%)$ \\
\hline Otros & $7(5,8 \%)$ \\
\hline \multicolumn{2}{|l|}{ Tipo } \\
\hline TT aislado & $39(32,5 \%)$ \\
\hline TT con lesiones extratorácicas & $81(67,5 \%)$ \\
\hline Politraumatismo & $76(63,3 \%)$ \\
\hline
\end{tabular}

TT: Traumatismo torácico.

un patrón "escalonado" característico. En el TT penetrante, por otro lado, existió un rápido incremento de la mortalidad en los primeros tres días, para luego mantenerse relativamente constante luego del día 15. Similares características se observaron en la mortalidad acumulada del TT con lesiones extratorácicas y el TT aislado.

Finalmente, la gran mayoría de los fallecidos correspondieron a TT contuso y con lesiones extratorácicas, los cuales aumentaron de forma sostenida desde el segundo día hasta la segunda semana desde 
Tabla 5. Principales lesiones en hospitalizados fallecidos con traumatismo torácico

\begin{tabular}{lc} 
Lesiones y/o hallazgos torácicos & \\
Hemotórax & $83(69,2 \%)$ \\
$\quad$ Hemotórax masivo & $17(14,2 \%)$ \\
Fracturas costales & $62(51,7 \%)$ \\
$\quad$ Tórax volante & $20(16,7 \%)$ \\
Neumotórax & $51(42,5 \%)$ \\
Contusión pulmonar & $46(38,3 \%)$ \\
Penetrante cardíaca & $28(23,3 \%)$ \\
Lesión de grandes vasos & $12(10,0 \%)$ \\
Lesión diafragmática & $9(7,5 \%)$ \\
Lesión del parénquima pulmonar & $8(6,7 \%)$ \\
Fractura esternal & $2(1,7 \%)$ \\
\hline Lesiones y/o hallazgos extratorácicos & \\
Craneoencefálicas & $45(37,5 \%)$ \\
Abdominales & $38(31,7 \%)$ \\
Extremidades & $32(26,7 \%)$ \\
Fractura de columna & $16(13,3 \%)$ \\
Fractura de pelvis & $14(11,7 \%)$ \\
Faciales & $8(6,7 \%)$ \\
Partes blandas & $7(5,8 \%)$ \\
Raquimedular & $5(4,2 \%)$ \\
\hline &
\end{tabular}

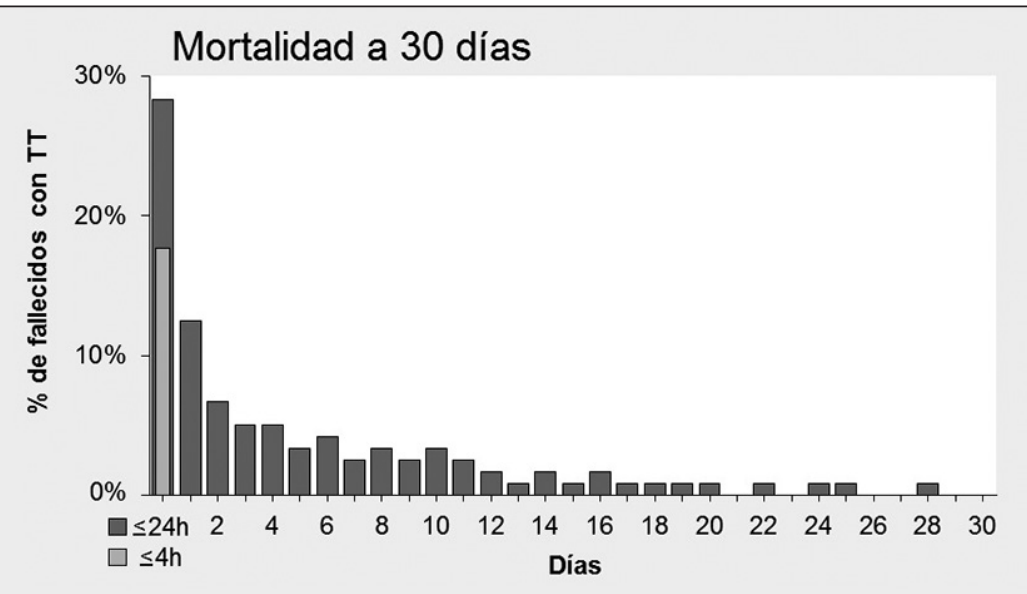

Figura 1. Distribución de la mortalidad a 30 días en hospitalizados fallecidos con traumatismo torácico (TT: traumatismo torácico).
Tabla 6. Causa atribuible de mortalidad y distribución temporal de la mortalidad en hospitalizados fallecidos con traumatismo torácico

\begin{tabular}{|c|c|}
\hline Total de fallecidos & $120(100 \%)$ \\
\hline \multicolumn{2}{|c|}{ Causa principal de muerte } \\
\hline Shock hipovolémico & $52(43,3 \%)$ \\
\hline SIRS/FOM & $37(30,8 \%)$ \\
\hline Sepsis & $14(11,7 \%)$ \\
\hline TEC grave & $13(10,8 \%)$ \\
\hline Otros & $4(3,4 \%)$ \\
\hline Mortalidad $\leq 7$ días & $79(100 \%)$ \\
\hline Shock hipovolémico & $52(65,8 \%)$ \\
\hline SIRS/FOM & $15(19,0 \%)$ \\
\hline TEC grave & $8(10,1 \%)$ \\
\hline Sepsis & $2(2,5 \%)$ \\
\hline Otros & $2(2,5 \%)$ \\
\hline Mortalidad a 8-30 días & $32(100 \%)$ \\
\hline SIRS/FOM & $18(56,3 \%)$ \\
\hline Sepsis & $7(21,9 \%)$ \\
\hline TEC grave & $5(15,6 \%)$ \\
\hline Otros & $2(6,2 \%)$ \\
\hline Mortalidad > 30 días & $9(100 \%)$ \\
\hline Sepsis & $5(55,6 \%)$ \\
\hline SIRS/FOM & $4(44,4 \%)$ \\
\hline
\end{tabular}

SIRS: síndrome de respuesta inflamatoria sistémica; FOM: falla orgánica múltiple; TEC: traumatismo encefalocraneano.

Tabla 7. Distribución temporal de la mortalidad e índices de gravedad del traumatismo en hospitalizados fallecidos con traumatismo torácico

\begin{tabular}{|lc|}
\hline Total de fallecidos & $120(100 \%)$ \\
Mortalidad $\leq 7$ días & $79(65,8 \%)$ \\
Mortalidad $\leq 4 \mathrm{~h}$ & $22(18,3 \%)$ \\
Mortalidad $\leq 24 \mathrm{~h}$ & $34(28,3 \%)$ \\
Mortalidad a $8-30$ días & $32(26,7 \%)$ \\
Mortalidad $>30$ días & $9(7,5 \%)$ \\
Índices de gravedad del traumatismo & \\
Injury Severity Score (ISS) & $27,8 \pm 13,3$ \\
Revised Trauma Score Triage (RTS-T) & $7,9 \pm 3,8$ \\
Trauma Injury Severity Score (TRISS) & 36,4 \\
\hline
\end{tabular}


Tabla 8. Distribución temporal de la mortalidad según clasificación del traumatismo en hospitalizados fallecidos con traumatismo torácico

\begin{tabular}{|lrcr|}
\hline & $\begin{array}{r}\text { TT contuso } \\
\mathbf{7 2 ( 1 0 0 \% )}\end{array}$ & $\begin{array}{c}\text { TT penetrante } \\
\mathbf{4 8 ( 1 0 0 \% )}\end{array}$ & p \\
Temporalidad & & & \\
Mortalidad $\leq 7$ días & $40(55,6 \%)$ & $39(81,3 \%)$ & $0,003^{*}$ \\
$\quad$ Mortalidad $\leq 4 \mathrm{~h}$ & $4(5,6 \%)$ & $18(37,5 \%)$ & $<0,001^{*}$ \\
Mortalidad $\leq 24 \mathrm{~h}$ & $12(16,7 \%)$ & $22(45,8 \%)$ & $<0,001^{*}$ \\
Mortalidad a 8-30 días & $24(33,3 \%)$ & $8(16,7 \%)$ & $0,043^{*}$ \\
Mortalidad $>30$ días & $8(11,1 \%)$ & $1(2,0 \%)$ & 0,137 \\
\hline
\end{tabular}

TT: Traumatismo torácico.

el ingreso, seguido del TT penetrante y aislado, los cuales presentaron una elevada mortalidad en los primeros días, particularmente en las primeras 4 y 24 h (Figura 5).

\section{Discusión}

En nuestra serie, las principales variables independientes asociadas a mortalidad tuvieron directa relación con el deterioro de los parámetros fisiológicos al momento del ingreso (RTS-T $<11$ ), la gravedad anatómica de las lesiones y la existencia de características que le otorgan mayor mortalidad al traumatismo,

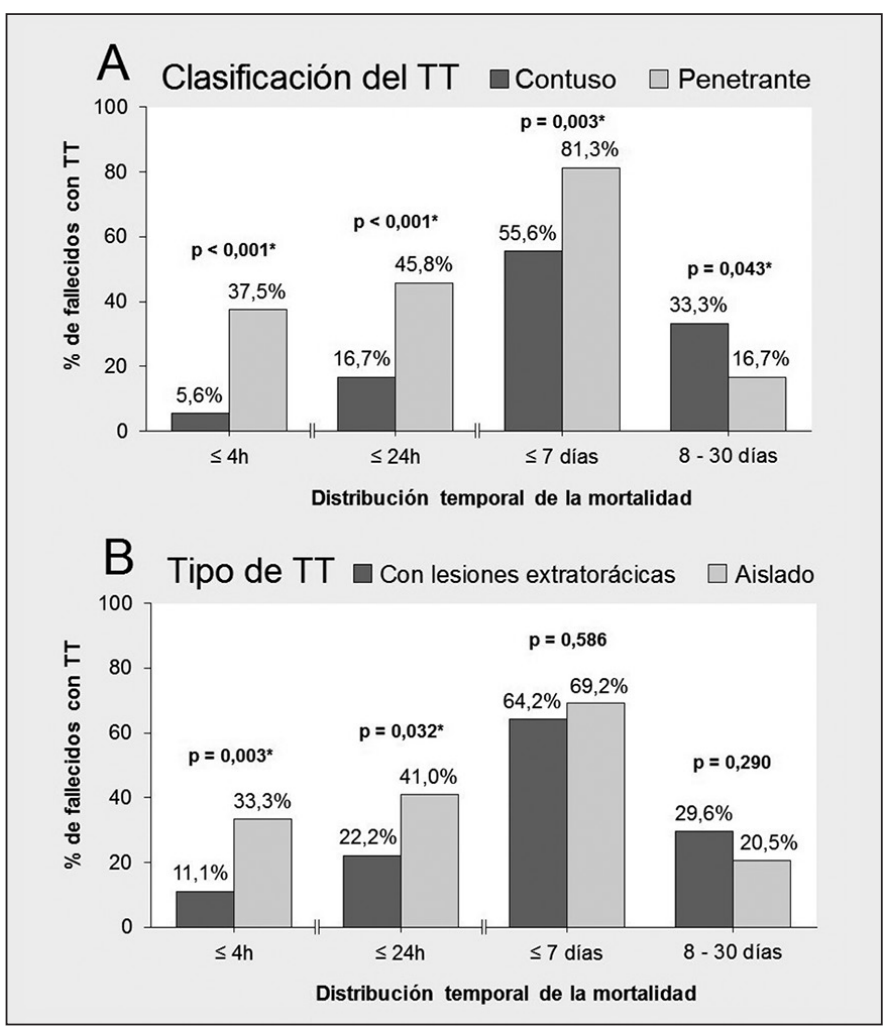

Figura 2. Distribución temporal de la mortalidad según clasificación y tipo del traumatismo en pacientes fallecidos con traumatismo torácico (TT: traumatismo torácico; *Estadísticamente significativo).

Tabla 9. Comparación de causa de muerte y distribución temporal de la mortalidad según tipo de traumatismo en pacientes fallecidos con traumatismo torácico

\begin{tabular}{|c|c|c|c|}
\hline & $\begin{array}{c}\text { TT con lesiones extratorácicas } \\
81(100 \%)\end{array}$ & $\begin{array}{l}\text { TT aislado } \\
39(100 \%)\end{array}$ & $\mathbf{p}$ \\
\hline $\begin{array}{l}\text { Causa de muerte } \\
\text { Shock hipovolémico } \\
\text { SIRS/FOM } \\
\text { Sepsis } \\
\text { TEC grave } \\
\text { Otros }\end{array}$ & $\begin{array}{c}27(33,3 \%) \\
30(37,0 \%) \\
9(11,1 \%) \\
13(16,1 \%) \\
2(2,5 \%)\end{array}$ & $\begin{array}{c}25(64,1 \%) \\
7(18,0 \%) \\
5(12,8 \%) \\
- \\
2(5,1 \%)\end{array}$ & $\begin{array}{c}0,001^{*} \\
0,034^{*} \\
0,769 \\
- \\
0,828\end{array}$ \\
\hline $\begin{array}{l}\text { Temporalidad } \\
\text { Mortalidad } \leq 7 \text { días } \\
\text { Mortalidad } \leq 4 \mathrm{~h} \\
\text { Mortalidad } \leq 24 \mathrm{~h} \\
\text { Mortalidad a } 8-30 \text { días } \\
\text { Mortalidad }>30 \text { días }\end{array}$ & $\begin{array}{c}52(64,2 \%) \\
9(11,1 \%) \\
18(22,2 \%) \\
24(29,6 \%) \\
5(6,2 \%)\end{array}$ & $\begin{array}{l}27(69,2 \%) \\
13(33,3 \%) \\
16(41,0 \%) \\
8(20,5 \%) \\
4(10,3 \%)\end{array}$ & $\begin{array}{c}0,586 \\
0,003 * \\
0,032^{*} \\
0,290 \\
0,670\end{array}$ \\
\hline $\begin{array}{l}\text { Índices de gravedad del traumatismo } \\
\text { Injury Severity Score (ISS) } \\
\text { Revised Trauma Score Triage (RTS-T) } \\
\text { Trauma Injury Severity Score (TRISS) }\end{array}$ & $\begin{array}{c}31,4 \pm 12,6^{* *} \\
7,9 \pm 3,8 \\
41,0\end{array}$ & $\begin{aligned} 19,2 & \pm 13,4 \\
7,8 & \pm 3,8 \\
33,8 & \end{aligned}$ & \\
\hline
\end{tabular}

TT: traumatismo torácico, SIRS: síndrome de respuesta inflamatoria sistémica; FOM: falla orgánica múltiple; TEC: traumatismo encefalocraneano. *Estadísticamente significativo; **En el subgrupo de TT con lesiones extratorácicas, el 95,1\% de las lesiones torácicas fueron graves o muy graves según ISS. 


\section{ARTÍCULO ORIGINAL}

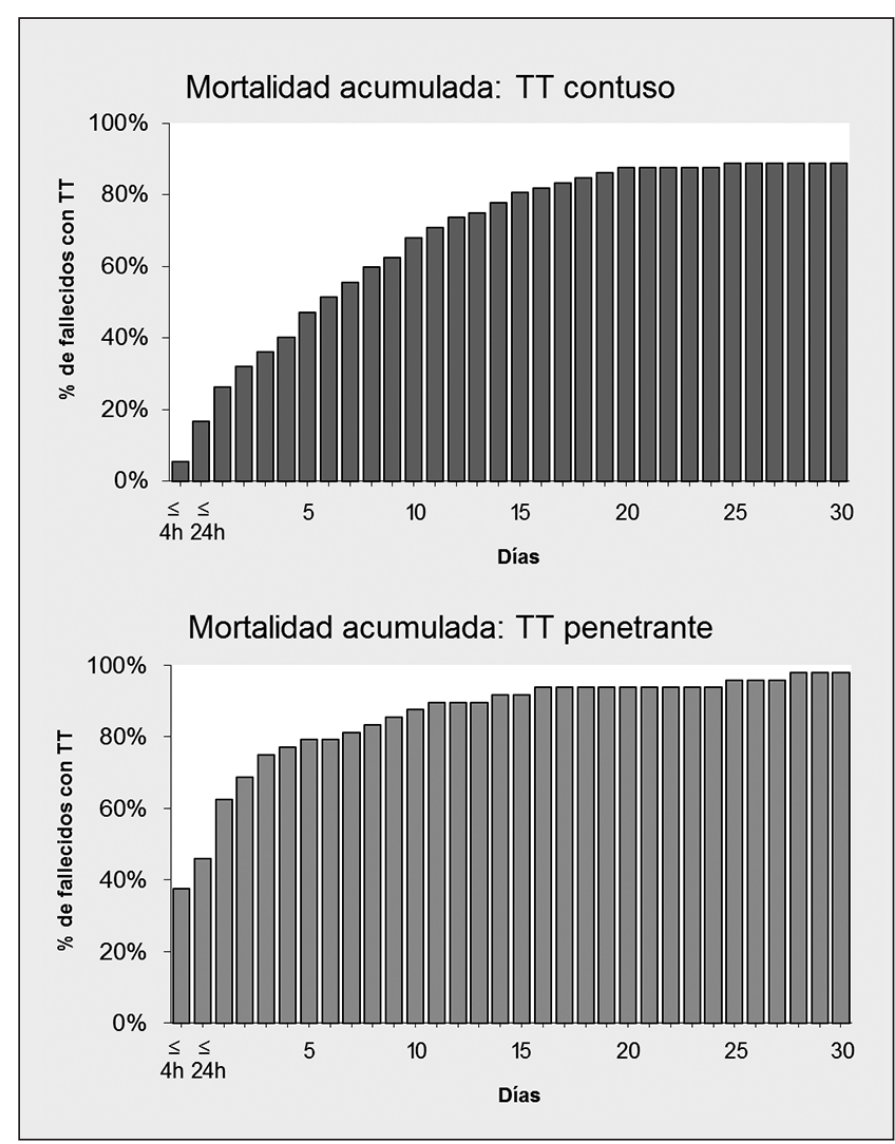

Figura 3. Mortalidad acumulada según clasificación del traumatismo en hospitalizados fallecidos con traumatismo torácico (TT: traumatismo torácico).

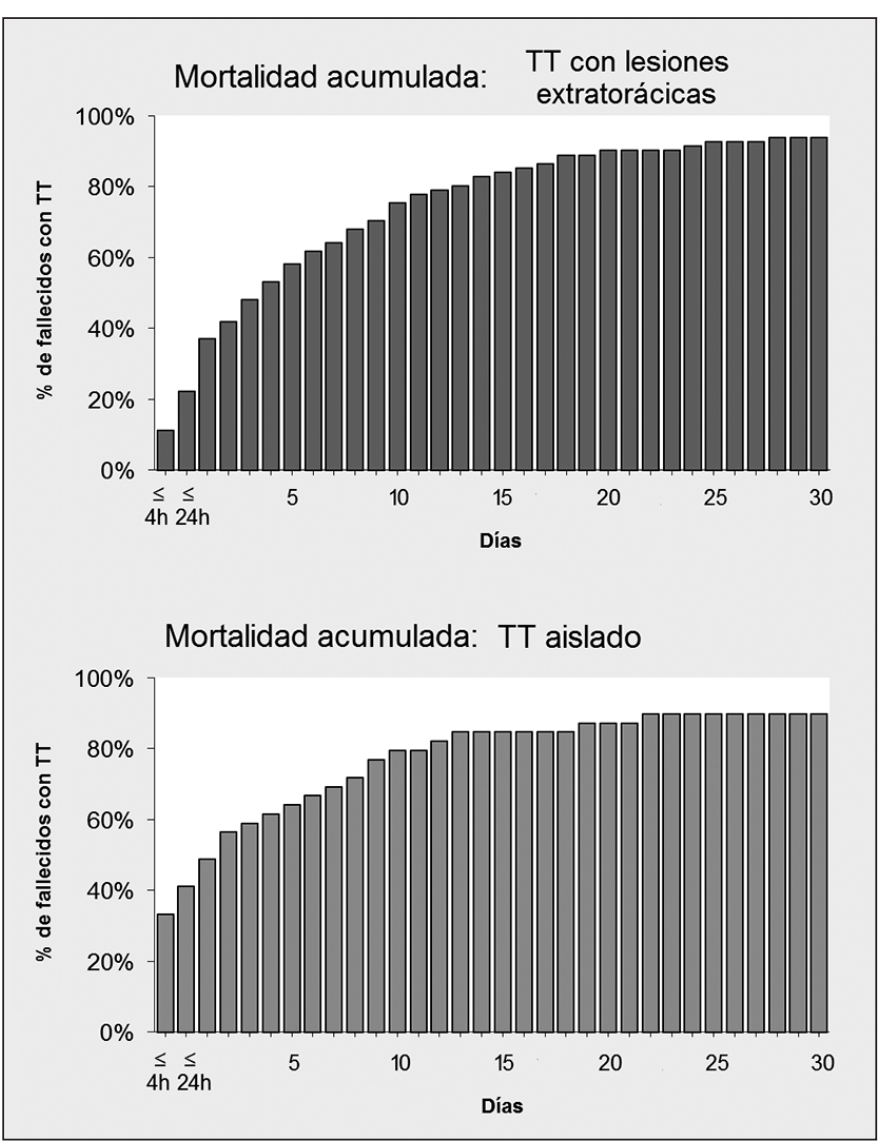

Figura 4. Mortalidad acumulada según tipo del traumatismo en hospitalizados fallecidos con traumatismo torácico (TT: traumatismo torácico).

como lo son el TT contuso, el politraumatismo, por arma de fuego y la asociación de éstas con el hemotórax masivo o el traumatismo penetrante cardíaco. Además, la mortalidad se concentró en las primeras horas luego del traumatismo, existiendo diferencias estadísticamente significativas según la causa de muerte, la clasificación o el tipo del TT y no se observó otro peak de la mortalidad luego de la primera semana.

Desde una perspectiva histórica, los principales conocimientos y avances en el tratamiento del TT han acontecido en contextos de guerra. En la Primera Guerra Mundial se describía una mortalidad del TT de aproximadamente el $50 \%$ y desde entonces se constató una disminución progresiva y sustancial de la mortalidad del TT en los conflictos bélicos siguientes, llegando a su menor registro en la Guerra de Vietnam, con valores cercanos al $5 \%{ }^{17-19}$. Esto se explica por las mejoras ocurridas durante la Segunda Guerra Mundial en: el entendimiento de la fisiopatología del TT, la optimización del Triage, la estandarización de la reanimación inicial avanzada y nuevas definiciones e indicaciones para la cirugía de urgencia en el TT, entre otros ${ }^{19,20}$.

Los últimos reportes de las características y la mortalidad

igura 5. Mortalidad acumulada global según clasificación y tipo del traumatismo en hospitalizados fallecidos con traumatismo torácico (TT: traumatismo torácico). 
del TT en zonas de guerra derivan de las experiencias en Iraq y Afganistán, donde se han observado cambios epidemiológicos en el agente y mecanismo del TT, con alta proporción de traumatismos por explosivos y heridas químicas, lo que ha provocado un aumento de la mortalidad, siendo actualmente cercana al $10 \%{ }^{21,22}$. Esto se explica porque existiría un aumento de las hemorragias incoercibles, lesiones de grandes vasos y hemotórax masivo a propósito de dichos agentes, por lo que se mantiene como un tópico de constante estudio y revisión ${ }^{23.24}$.

En zonas urbanas no militarizadas, en cambio, la mortalidad del TT presenta amplias variaciones entre diferentes series, lo que depende principalmente de las variables sociodemográficas de la población estudiada. Así, en casuísticas donde predomina el TT contuso debido a accidentes de tránsito de alta energía, la mortalidad del TT puede superar el 10\%$15 \%$, mientras que, en zonas con alta prevalencia de agresiones por arma blanca, la mortalidad global es generalmente menor al 5\% $\%^{25-28}$.

Diferentes estudios han analizado los factores que se asocian a complicaciones y mortalidad en hospitalizados con TT, y la mayoría de ellos concuerda en que el deterioro hemodinámico al momento del ingreso, la asociación con lesiones extratorácicas y la necesidad de cuidados intensivos son las principales variables asociadas a mortalidad en $\mathrm{TT}^{7,29-33}$. En este contexto, nuestros resultados fueron semejantes a lo descrito internacionalmente.

Battle y cols., en una revisión sistemática y metaanálisis de los factores predictores de mortalidad en TT contuso encontraron que el riesgo de mortalidad aumenta significativamente desde los 65 años, cuando se asocia a tres o más fracturas costales y cuando existe enfermedad cardiopulmonar preexistente $^{31}$. Además, Pehlivanlar y cols., estimaron que la edad fue un factor independiente de mortalidad, agregándose 1,025 veces más riesgo de fallecer por cada año adicional desde los 18 años de edad y que, entre los pacientes que requirieron cuidados intensivos, la asociación con TEC fue el factor de riesgo más importante 7 .

Existen escasas publicaciones que analicen los factores asociados a mortalidad del TT penetrante de forma general, sin embargo, se cuenta con la evidencia derivada del estudio del traumatismo penetrante cardíaco y del hemotórax masivo ${ }^{34-36}$. En la experiencia recientemente publicada de nuestro grupo, el deterioro hemodinámico consistente en paro cardiorrespiratorio y shock al momento del ingreso son los factores más importantes que determinan la mortalidad de estos $\mathrm{TT}^{36,37}$. Estos hallazgos son similares a lo reportado por otros autores ${ }^{34-36}$.
La principal causa de muerte en nuestra serie correspondió al shock hipovolémico, lo que es concordante con otras series específicas de TT. No obstante, cuando se analizan las causas de muerte del traumatismo en general, se observa que aproximadamente entre el $40 \%-60 \%$ se atribuyen a lesiones graves del sistema nervioso central, fundamentalmente debido a accidentes de tránsito o caídas de altura $^{30,38,39}$. En nuestra serie, aproximadamente el $10 \%$ de los fallecidos con TT presentaron un TEC grave como causa principal a la cual se le atribuyó la mortalidad.

Existieron diferencias en las principales causas de muerte según la distribución temporal de la mortalidad, siendo más frecuente la causa hemorrágica en los primeros siete días luego del traumatismo, el SIRS/FOM entre la segunda y cuarta semana y la sepsis después de 30 días. Esto es comparable a lo descrito por Belamy, quien postuló que las principales causas de muerte del traumatismo (exanguinación, TEC y FOM) presentaban patrones de distribución temporal característicos y predecibles, siendo importante la exanguinación en las primeras 24-36 h, el TEC en los primeros cinco días y la FOM a partir del segundo día de hospitalización ${ }^{40}$.

La distribución temporal de la mortalidad por traumatismos ha sido extensamente estudiada desde que Trunkey postulara el concepto de distribución trimodal en $1982^{41}$. En ese entonces, observó que aproximadamente el $45 \%$ de los fallecimientos ocurrían en la primera hora después del traumatismo debido a lesiones incompatibles con la vida. Un segundo peak (35\%) acontecía antes de las $4 \mathrm{~h}$ provocado por lesiones graves del sistema nervioso central o exanguinación $\mathrm{y}$, finalmente, un tercer peak de mortalidad $(20 \%)$ luego de la primera semana secundario a complicaciones tardías del traumatismo ${ }^{41}$.

En la actualidad, numerosos estudios a nivel mundial no han logrado replicar la distribución trimodal debido a los cambios ocurridos en la temporalidad de la muerte producto de la optimización de la atención prehospitalaria, el desarrollo del soporte vital avanzado y mejoras en las técnicas quirúrgicas, entre otros ${ }^{38,42-45}$. En nuestra serie no se observaron peak de mortalidad después del primer día.

Sin embargo, en nuestra serie sí fue posible observar diferencias en la distribución temporal de la mortalidad acumulada cuando se comparó según clasificación y tipo del TT. En los primeros días y particularmente en las primeras 4 y $24 \mathrm{~h}$ se encontró un rápido incremento de la mortalidad del TT penetrante y aislado, provocado por shock hipovolémico, debido principalmente a traumatismo 
penetrante cardíaco o lesión de grandes vasos. Por otro lado, el subgrupo con mayor mortalidad fue el TT contuso y con lesiones asociadas, ocurriendo de forma más diferida, secundario a las complicaciones del politraumatismo (SIRS/FOM o sepsis) y debido principalmente a accidentes de tránsito.

Una de las limitaciones de nuestro estudio es que incluyó únicamente a fallecidos con TT que recibieron atención hospitalaria, por lo que no refleja el número real de fallecidos con TT, ya que desconocemos el porcentaje de estos que fallecen en el sitio del traumatismo. Además, nuestra serie solo describe la mortalidad en hospitalizados con TT y no la provocada específicamente por un TT, debido a que en más de un $10 \%$ la causa de muerte principal se atribuyó a una lesión extratorácica grave. Sin embargo, en casi la totalidad de los fallecidos observamos que la lesión torácica fue grave o muy grave según ISS, por lo que consideramos que contribuyó de manera significativa en el desenlace fatal de estos pacientes.

En conclusión, las principales variables indepen- dientes asociadas a mortalidad en hospitalizados con TT fueron: deterioro fisiológico al ingreso (RTS-T $<11$ ), hemotórax masivo y TT por arma de fuego. Predominó el shock hipovolémico y el SIRS/FOM como causas de muerte, encontrándose diferencias en la distribución temporal. Finalmente, el TT contuso con lesiones extratorácicas fue el que más contribuyó a la mortalidad de la serie, mientras que el TT penetrante y aislado presentó muertes más precoces.

\section{Responsabilidades éticas}

Protección de personas y animales. Los autores declaran que para esta investigación no se han realizado experimentos en seres humanos ni en animales.

Confidencialidad de los datos. Los autores declaran que en este artículo no aparecen datos de pacientes.

Conflictos de interés: no hay.

\section{Bibliografía}

1. Murray CJ, López AD. Mortality by cause for eight regions of the world: Global Burden of Disease Study. Lancet 1997;349:1269-81.

2. Demirhan R, Onan B, Oz K, Halezeroglu S. Comprehensive analysis of 4205 patients with chest trauma: a 10-year experience. Interact Cardiovasc Thorac Surg. 2009;9:450-3.

3. Hodgson NF, Stewart TC, Girotti MJ. Autopsies and death certification in deaths due to blunt trauma: what are we missing?: Can J Surg. 2000;43:130-6.

4. González R, Riquelme A, Fuentes A, Saldías R, Reyes R, Seguel E, et al. Traumatismo torácico: caracterización de hospitalizaciones durante tres décadas. Rev Med Chile 2018;146:196-205.

5. O'Connor JV, Adamski J. The diagnosis and treatment of non-cardicac thoracic trauma. J R Army Med Corps 2010;156:514.

6. Shackford SR, Mackersie RC, Holbrook TL, Davis JW, Hollingsworth-Fridlund P, Hoyt DB, et al. The epidemiology of traumatic death. A population-based analysis. Arch Surg. 1993;128:571-5.

7. Pehlivanlar M, Oguzhan A, Aksoy Í,
Aydin, Ülger F. Prognostic evaluation of cases with thoracic trauma admitted to the intensive care unit: 10 -year clinical outcomes. Ulus Trauma Acil Cerrahi Derg. 2019;25:46-54.

8. Baker SP, O'Neill B, Haddon W, Long W. The Injury Severity Score: a method for describing patients with multiple injuries and evaluating emergency care. J Trauma 1974;14:187-96.

9. Champion H, Sacco W, Copes W, Gann D, Gennarelli T, Flanagan M. A revision of the Trauma Score. J Trauma 1989;29:6239.

10. Boyd C, Tolson M, Copes W. Evaluating trauma care: the TRISS method. Trauma Score and Injury Severity Score. J Trauma 1987;27:370-8.

11. Freixinet J, Beltrán J, Rodríguez P, Juliá G, Hussein M, Gil R, et al. Indicators of Severity in Chest Trauma. Arch Bronconeumol. 2008:44:257-62.

12. Mica L, Rufibach K, Keel M, Trentz O. The risk of early mortality of polytrauma patients associated to ISS, NISS, APACHE II values and prothrombin time. J Trauma Manag Outcomes 2013;7:6.

13. Informe mundial sobre el envejecimiento y salud. En: Organización Mundial de la Salud. 2015. WHO/FWC/ALC/15.01.
14. González R, Barra S, Riquelme A, Reyes R, Alarcón F, Seguel E, et al. Traumatismo torácico: análisis de hospitalizaciones según grupo etario. Rev Cir. 2021;73:41019.

15. Rangel-Frausto MS, Pittet, Costigan M, Hwang T, Davis CS, Wenzel RP. The natural history of the Systemic Inflammatory Response Syndrome (SIRS). A prospective study. JAMA 1995;273:117-23.

16. Carrico CJ, Meakins JL, Marshall JC, Fry D, Maier RV. Multiple-OrganFailure Syndrome. Arch Surg. 1986;121:196-208.

17. Kuckelman J, Cuadrado D, Martin M. Thoracic trauma: a combat and military perspective. Curr Trauma Rep 2018;4:7787.

18. Mansky R, Scher C. Thoracic trauma in military settings: a review of current practices and recommendations. Curr Opin Anesthesiol. 2019;32:227-33.

19. McNamara J, Messersmith J, Dunn R, Molot M, Stremple J. Thoracic injuries in combat: casualities in Vietnam. Ann Thorac Surg. 1970;10:389-401.

20. Senenayake EL, Poon H, Graham TR, Midwinter MJ. UK specialist cardiothoracic management of thoracic 
injuries in military casualities sustained in the wars in Iraq and Afghanistan. Eur J Cardiothorac Surg. 2014;45:e202-7.

21. Keneally RJ, Szpisjak DF, Hoffmann PJ, Park EJ, Albergo MS. Vital signs and physiologic derangement in patients with thoracic trauma in Iraq and Afghanistan. Mil Med 2017;182:e1881-4.

22. Stern CA, Stockinger ZT, Gurney JM. Combat thoracic surgery in Iraq and Afghanistan 2002-2016. J Trauma Acute Care Surg. 2020;89:551-7.

23. Poon H, Morrison JJ, Apodaca AN, Khan MA, Garner JP. The UK military experience of thoracic Injury in the wars in Iraq and Afghanistan; Injury 2013;44:1165-70.

24. Keneally R, Szpisjak D. Thoracic trauma in Iraq and Afghanistan. J Trauma Acute Care Surg 2013;74:1292-7.

25. El-Din ML, Nashy MR, Meshhal MT. Blunt vs. Penetrating chest trauma in term of the outcome in Menoufia University Hospital. Menoufia Med J. 2018;31:133-9.

26. Grubmüller M, Kerschbaum M, Diepold E, Angerpointner K, Nerlich M, Ernstberger A. Severe thoracic traumastill and independent predictor for death in multiple injured patients?. Scand J Trauma Resusc Emerg Med. 2018;26:6.

27. González R, Riquelme A, Toloza C, Reyes R, Seguel E, Stockins A, et al. Traumatismo torácico contuso. Rev Chil Enferm Respir. 2019;35:96-103.

28. González R, Riquelme A, Toloza C, Reyes R, Seguel E, Stockins A, et al. Traumatismo torácico por arma blanca. Rev Cir. 2020;72:137-43.

29. Lefering R, Paffrath T, Bouamra O, Coats TJ, Woodford M, Jenks T, et al. Epidemiology of in-hospital trauma deaths. Eur J Trauma Emerg Surg. 2012;38:3-9.
30. Pfeifer R, Teuben M, Andruszkow H, Barkatali BM, Pape HC. Mortality patterns in patients with multiple trauma: A systematic review of autopsy studies. PLoS One 2016;11(2):e0148844. doi: 10.1371/journal.pone.0148844.

31. Battle CE, Hutchings H, Evans PA. Risk factors that predict mortality in patients with blunt chest wall trauma: A systematic review and meta-analysis. Injury 2012;43:8-17.

32. González R, Riquelme A, Ávalos Á, Reyes R, Seguel E, Stockins A, et al Traumatismo torácico por arma de fuego. Rev Cir. 2020;72:293-300.

33. González R, Riquelme A, Fuentes A, Canales J, Seguel E, Stockins A, et al. Traumatismo penetrante cardíaco: caracterización, resultados inmediatos $\mathrm{y}$ variables asociadas a morbilidad y mortalidad en pacientes operados. Rev Cir. 2019;71:245-52.

34. Asensio JA, Ogun OA, Petrone P, Pérez-Alonso AJ, Wagner M, Bertellotti $\mathrm{R}$, et al. Penetrating cardiac injuries: predictive model for outcomes based on 2016 patients from the National Trauma Data Bank. Eur J Trauma Emerg Surg. 2018;44:835-41.

35. Hirshberg A, Wall MJ Jr., Allen MK, Mattox KL. Double jeopardy: thoracoabdominal injuries requiring surgical intervention in both chest and abdomen. J Trauma 1995;39:225-9.

36. González R, Riquelme A, Toloza C, Reyes R, Seguel E, Stockins A, et al. Hemotórax masivo por traumatismo torácico en pacientes tratados quirúrgicamente. Rev Cir. 2020;72:434-40.

37. González R, Alarcón F, Riquelme A, Reyes R, Barra S, Seguel E, et al. Evolución del traumatismo penetrante cardíaco operado. Rev Cir. 2021; 73: 401-9.

38. Lansink KWW, Gunning AC, Leenen
LPH. Cause of death and time of death distribution of trauma patients in a Leves I trauma centre in the Netherlands. Eur J Trauma Emerg Surg. 2013;39:375-83.

39. Soreide K, Krüger AJ, Vardal AL, Ellingsen CL, Soreide E, Lossius HM. Epidemiology and contemporary patterns of trauma deaths: Changing place, similar pace, older face. World J Surg. 2017;31:2092-103.

40. Belamy RF. The causes of death in conventional land warface: implications for combat casualty care research. Mil Me. 1984;149:55-62.

41. Trunkey DD. Trauma. Accidental and intentional injuries account for more years in the U.S. tan cancer and heart disease. Among the prescribed remedies are improved preventive efforts, speedier surgery and further research. Sci Am. 1983;249:28-35.

42. Knegt CK, Meylaerts SAG, Leenen LPH. Applicability of the trimodal distribution of trauma deaths in a Level I trauma centre in the Netherlands with population of mainly blunt trauma. Injury 2008;39:933-1000

43. Rauf R, Von Matthey F, Croenlein M, Zyskowski M, Van Griensven M, Biberthaler $\mathrm{P}$, et al. Changes in the temporal distribution of in-hospital mortality in severely injured patients-An analysis of the Trauma Register DGU. PLoS One 2019;14:e0212095.

44. Pang JM, Civil I, Ng A, Adams D, Koelmeyer T. I the trimodal pattern of death after trauma a dated concept in the 21 st century? Trauma death in Auckland 2004. Injury 2008;39:102-6.

45. Gunst M, Ghaemmaghami V, Gruszecki A, Urban J, Frankel H, Shafi S. Changing epidemiology of trauma deaths leads to a bimodal distribution. Proc Bayl Univ Med Cent. 2010;23:349-54. 